\title{
Post-operative agitation in adults, factors, possible mechanisms and prevention.
}

\author{
Hazem E Elsersy* \\ Department of Medicine, Menoufya University, Egypt
}

*Correspondence to: Hazem Ezzat Elsersy, Lecturer of Anesthesia, Faculty of Anesthesiology, Menofia University, Address: Zaki Shabanah Street, Alzanati tower, Shebin Alkom, Menofia, Egypt, Tel: 00201091096655; E-mail: hazelsersy@hotmail.com

Received date: October 7, 2017; Accepted date: October 8, 2017; Published date: October 16, 2017

Citation: Elsery EH. Post-operative agitation in adults, factors, possible mechanisms and prevention. Anaesthesiol Clin Sci Res. 2017;1(1):1-3.

Copyright: (C) 2017 Elsery HE. This is an open-access article distributed under the terms of the Creative Commons Attribution License, which permits unrestricted use, distribution, and reproduction in any medium, provided the original author and source are credited.

\section{Introduction}

Postoperative agitation (POA) although short-lived is potentially harmful to the patient and the recovery staff [1]. In the postoperative care unit, an agitated patient requires more nurses to control his abnormal movement and apply restrains that could result in bruises of his extremities. In the postoperative care unit, we noticed that agitated patients remove venous and arterial catheters, nasal packs, oxygen masks and endotracheal tubes leading to bleeding and hypoxia.

Post-operative agitation is a well-documented phenomenon in children recovering from sevoflurane anesthesia [2]. However, in adults, few reports have highlighted the occurrence of this phenomenon [3,4]. The exact trigger for this complication remains to be determined, however, many factors play a crucial role in revealing POA. While an alert, calm patient is the ideal recovery outcome, the occurrence of excitation, excessive motor movements and unexplained sounds is not an uncommon scenario following nasal surgery in adults. Several factors have been incriminated to provoke agitations in adults; pain, hypoxia, type of operation, too rapid emergence from anesthesia, sedatives like benzodiazepines [5-7].

\section{Post-operative agitation following nasal surgery}

The incidence of postoperative agitation following nasal surgery is relatively high [8]. The exact mechanism is Unknown. However, Kim et al. [3] reported that the occurrence of POA could be as high as $55.4 \%$ and the presence of nasal pack is likely to be the main trigger of agitation. In our latest study [8], we reported an incidence of $68 \%$ following nasal surgery. We highlighted several factors that may increase the prevalence of POA; Male gender, young age, smoking, postoperative pain and premedication with atropine and Phenergan. Yu et al. [9] reported a 55\% incidence of POA after nasal surgery. They demonstrated that Doxapram administration, pain, and presence of a tracheal tube and or a urinary catheter appear to be the most important causes of postoperative agitation. Similarly, pain, urinary catheters, and tracheal tubes were also reported by Kim et al. [3] as risk factors for POA following nasal surgery. The presence of nasal pack as a trigger to POA remains debatable.

\section{Possible mechanistic interactions (A hypothesis)}

The mechanism by which a patient develops agitation remains unclear. The phenomenon of POA became more apparent since the advent of rapid emergence inhalational anesthetics; sevoflurane and desflurane [10]. Inhalational induction of anesthesia occurs in four stages according to Guedel's classification; stage two is characterized by patient excitation that mimics an agitated patient who exhibits a marked excited and delirious activity. This excitation becomes manifest if the patient enters into light sedation stage then it dissipates completely if the patient enters into a deep stage which is the surgical anesthesia state. Benzodiazepines have been shown to increase excitation and agitation! [11,12] How can a sedative drug cause sedation and excitation in the meantime? These observations and questions raise the possibility that an agitated patient is not in the complete awake mode during this agitation. What enforces this assumption is that the patient during agitation is not responding to commands and behaves like a drunken person.

Therefore POA state may be due to incomplete recovery because sevoflurane closure results in a rapid emergence from the inhalational component, while the effect of the other anesthetics may be still there. In our last report [8], premedicated patients showed a substantially increased agitation and excitation this effect might be due to the remaining effects of the premedication after dissipation of the sevoflurane effect. Some factors could enforce POA, for instance, young age, and male gender has a higher frequency of agitation. The striking difference between a male and a female is mainly in the estrogen and testosterone hormones. Interestingly testosterone has been shown to steadily decline with advancing age [13], so it is possible that the increased testosterone in young males could be a factor in increased agitation. Smokers showed more frequent agitations, the matter that points to nicotine a central nervous system stimulant as a contributing factor. Nicotine withdrawal is accompanied by excitation and negative emotional state [14], so it could be the 
Citation: Hazem E Elsersy. Post-operative agitation in adults, factors, possible mechanisms and prevention. Anaesthesiol Clin Sci Res 2017;1(1):2.

abstinence during the fasting period that results in this excitation. The presence of urinary catheters and tracheal tubes increase the sense of discomfort. The reaction to this discomfort may be augmented in the light anesthesia state resulting in agony and agitation. Pain is a consistent cause of postoperative agitation $[15,16]$. While an awake patient can express his pain by telling I have pain or I have a severe pain conversely, a patient in the light anesthesia stage is confused and cannot respond to the command, so he expresses his pain in the form of agitation.

In summary, we propose that two events should interact to provoke postoperative agitation; the first is mental confusion manifested as incomplete anesthetic recovery, the second is the perception of a discomfort such as pain, irritation by catheter, tubing, restraints, nicotine or drug withdrawal.

\section{Agitation or delirium?}

Although both agitation and delirium are characterized by patient excitation and confusion both are different in their course, population and progress. Agitation is like a tornado; it hits rapidly but dissipates quickly. Agitation is short-lived while delirium lasts for long periods [17]. Agitation is more present in children and young age while delirium is manifest in the geriatric population. Agitation is benign whereas delirium is far from benign and may be fatal. Delirium occurs due to several factors that appear unrelated for example, hepatic encephalopathy, renal failure, some medications like anticholinergics, anti-histaminics, benzodiazepines, following cardiac surgery. For delirium to occur a state and a stimulus have to be present; a state of mental cloudiness that is probably due to a neurotoxin like ammonia in liver failure, alcohol intoxication drug withdrawal, Wernicke's encephalopathy, hypertensive encephalopathy, hypoglycemia, hypoperfusion, hypoxemia, intracranial hemorrhage, encephaliti and side effects of medications [18].An irritative stimulus such as tracheal tubes, restraints or catheters in an ICU admitted patient.

\section{Prevention of POA following nasal surgery}

Post-operative agitation could be prevented or minimized if the precipitating factors are avoided. For preoperative sedation, we can use dexmedetomidine instead of benzodiazepines as the former has been shown to decrease the incidence of agitation following nasal surgery [3]. Substitution of atropine by glycopyrrolate could be of help as the latter does not cross the blood-brain barrier. We have reported that magnesium sulfate decreases postoperative agitation following nasal surgery [19]. Hypotensive anesthesia is commonly used with nasal surgery to reduce bleeding and improve the surgical field, So for hypotensive anesthesia, it is prudent to use magnesium sulfate alone or combined with another hypotensive to reduce postoperative agitation. Particular attention should be given to the high-risk group of patients such as young age, males, and smokers. Finally, adequate control of postoperative pain by multimodal analgesic approach could be of help towards a smoother recovery with a calm, alert patient.

\section{References}

1. Rose DK. Recovery room problems or problems in the PACU. Can J Anaesth. 1996;43:116-28.

2. Veyckemans F. Excitation phenomena during sevoflurane anaesthesia in children. Curr Opin Anaesthesiol. 2001;14:339-43.

3. Kim SY, Kim JM, Lee JH, et al. Efficacy of intraoperative dexmedetomidine infusion on emergence agitation and quality of recovery after nasal surgery. $\mathrm{Br} \mathrm{J}$ Anaesth. 111(2):222-8.

4. Daihua Yu, Wei Chai, Xude Sun, et al. Emergence agitation in adults: Risk factors in 2,000 patients. Can $\mathbf{J}$ Anesth. 2010;.57(9):843-848.

5. Marcantonio ER, Juarez G, Goldman L, et al. The relationship of post-operative delirium with psychoactive medications. JAMA. 1994;272:1518-22.

6. Marcantonio E, Ta T, Duthie E, et al. Delirium severity and psychomotor types: Their relationship with outcomes after hip fracture repair. J AmGeriatr Soc. 2002; 50:850-7.

7. Jo“ hr M. Excitation following sevoflurane: A problem in pediatric anesthesia? Anaesthesist. 199948:917-8.

8. Elsersy HE, Ahmed AA. Factors affecting postoperative agitation in adults following functional endoscopic sinus surgery: A randomized, double-blinded controlled trial. Anaesth Crit Care Med J. 2017;2(2):000123.

9. Yu D, Chai W, Sun X, et al. Emergence agitation in adults: Risk factors in 2000 patients. Can J Anaesth. 2010;57:843-8.

10. Locatelli BG, Ingelmo PM, EmreSL, et al. Emergence delirium in children: A comparison of sevoflurane and desflurane anesthesia using the pediatric anesthesia emergence delirium scale. Pediatr Anesth. 2013;23:301-8.

11. Marcantonio ER, Juarez G, Goldman L, et al. The relationship of post-operative delirium with psychoactive medications. 1994;16:1518-22.

12. Pandharipande P, Shintani A, Peterson J, et al. Lorazepam is an independent risk factor for transitioning to delirium in intensive care unit patients. Anesthesiology. 2006;104(1):21-6.

13. Travison TG, Araujo AB, Kupelian V, et al. The relative contributions of aging, health and lifestyle factors to serum testosterone decline in men. J Clin Endocrinol Metab. 2007;92(2):549-55.

14. Benowitz NL Pharmacology of nicotine: Addiction, smoking-induced disease and therapeutics. Annu Rev Pharmacol Toxicol. 2009;49:57-71.

15. Lynch EP, Lazor MA, Gellis JE, et al. The impact of postoperative pain on the development of postoperative delirium. Anesth Analg 1998; 86:781-785.

16. Veyckemans F. Excitation phenomena during sevoflurane anesthesia in children. Curr Opin Anaesthesiol. 2001;14:339-43.

17. Rudolph JL, Marcantonio ER: postoperative delirium acute change with long-term implications. Anesth Analg. 2011;112:1202-11. 
18. Cassem NH, Murray GB, Lafayette JM, et al. Delirious patients. The MGH Handbook of General Hospital Psychiatry, 5th Edition. Edited by Stern TA, Fricchione GL, Cassem NH, et al. St. Louis, Mosby. 2004;119-34.

19. Elsersy HE, Metyas MC, Elfeky HA, et al. Intraoperative magnesium sulphate decreases agitation and pain in patients undergoing functional endoscopic surgery: A randomised double-blind study. Eur J Anaesthesiol. 2017;34(10):658-64. 